\title{
Analysis of the influence of the complex structure of clay hollow bricks on the values of electric field intensity by using the FDTD method
}

\author{
AgnieszKa Choroszucho \\ Faculty of Electrical Engineering, Biatystok University of Technology \\ Wiejska 45D, 15-351 Biatystok, Poland \\ e-mail: a.choroszucho@pb.edu.pl
}

(Received: 13.04.2016, revised: 18.10.2016)

\begin{abstract}
The study presents the analysis of the effects occurring at the propagation of electromagnetic waves within an area containing non-ideal, non-homogenous and absorbing dielectric. The analysed models are connected with housing constructions and include single and double-layered walls made of clay hollow bricks. The influence of the size of holes, the contained clay mass percentage and conductivity of brick on the distribution of electric field is presented. Double-layered wall causes more heterogeneity in distribution of electric field and numerous maxima and minima to compare with singlelayered construction. The presented results refer to the electromagnetic field generated by a wireless communication system (Wi-Fi), operating within the standard frequencies $(2.4 \mathrm{GHz}$ and $5 \mathrm{GHz})$. A FDTD method was used to the analysis of electric field distribution. Also in this paper all formulations of difference method (FDTD) is presented. The possibilities of modifying the described method are indicated too. The obtained values of electric field intensity allow to determining the attenuation coefficient for different variants of the walls. Detailed analysis of influence of different types of building construction will make it possible to better understand the wave phenomena and counteract local fading at planning of wireless networks systems.
\end{abstract}

Key words: building materials, electromagnetic waves propagation, Finite-Difference Time-Domain method (FDTD), wireless communication systems

\section{Introduction}

The detailed analysis of the phenomena connected with the influence of various building materials on the electromagnetic field distribution has been the subject of a number of studies for example [1-4]. The using of wireless communication systems (e.g. Wi-Fi) it must take into account some effects that may disturb the quality of data transmission, e.g. complex building construction. The typical constructions contain different types of brick (ceramics). The one of the aims of these investigations is a comparison of the influence of the size of holes inside the brick on the distribution of an electromagnetic field in the area close to the brick wall. 
In this paper the analysis of influence of changes of brick conductivity on the values of electric field was shown. The reason of this research was the fact of using different electrical parameters of brick by others authors [1,5]. For example, at $f=2.4 \mathrm{GHz}$ and $f=5 \mathrm{GHz}$ the range of conductivity of a brick material is greatly wide $(\sigma \in\langle 0.0174,0.12\rangle \mathrm{S} / \mathrm{m})$ and even $\sigma=0.244 \mathrm{~S} / \mathrm{m}[5]$.

\section{Mathematical model}

To determine the distribution of an electromagnetic field in the analysed model, the Finite-Difference Time-Domain method (FDTD) was used [6-8]. It is useful in calculating electromagnetic fields changing in time at the range of high frequencies and broadband signals. The FDTD method is based on Maxwell's curl equations in time and space [6-9]:

$$
\begin{gathered}
\nabla \times \boldsymbol{E}=-\frac{\partial \boldsymbol{B}}{\partial t}, \\
\nabla \times \boldsymbol{H}=\boldsymbol{J}_{P}+\boldsymbol{J}_{D}+\boldsymbol{J}_{I}, \\
\nabla \cdot \boldsymbol{D}=\rho, \\
\nabla \cdot \boldsymbol{B}=0, \\
\nabla \cdot \boldsymbol{J}=-\frac{\partial \rho}{\partial t},
\end{gathered}
$$

where: $\boldsymbol{E}$ is an electric field (vector), $\boldsymbol{H}$ is a magnetic field (vector). The sources of these fields are electric charges and electric currents, which can be expressed as local densities, namely charge density $\rho$ and current density $\boldsymbol{J}$.

The density of conduction current $\boldsymbol{J}_{P}$ and the density of displacement current $\boldsymbol{J}_{D}$ were described by dependences

$$
\begin{aligned}
& \boldsymbol{J}_{P}=\sigma \boldsymbol{E}, \\
& \boldsymbol{J}_{D}=\frac{\partial \boldsymbol{D}}{\partial t},
\end{aligned}
$$

whereas $\boldsymbol{J}_{I}$ is the significant vector of density of current which force the field.

The FDTD method allows the analysis of complex structures, in which every material has a corresponding electrical property material. Description issues by Eqs. (1)-(5) allows to determine the field distribution in the nonstacionary state and also steady state in the appearing of time-varying field excitations.

The full wordings of the discussed issues are described in the field of three-dimensionality. Due to the specific of building structures, the regularity of their geometry typing in a Cartesian coordinate system, adopted discretisation of Maxwell's equations in this reference frame. After applying a decomposition, Eqs. (1)-(2) are presented in the form of six conjugate differential equations of the first order, that describe the electric and magnetic field components [6-8] 


$$
\begin{gathered}
\frac{\partial E_{x}}{\partial t}=\frac{1}{\varepsilon} \cdot\left(\frac{\partial H_{z}}{\partial y}-\frac{\partial H_{y}}{\partial z}-\sigma E_{x}\right), \\
\frac{\partial E_{y}}{\partial t}=\frac{1}{\varepsilon} \cdot\left(\frac{\partial H_{x}}{\partial z}-\frac{\partial H_{z}}{\partial x}-\sigma E_{y}\right), \\
\frac{\partial E_{z}}{\partial t}=\frac{1}{\varepsilon} \cdot\left(\frac{\partial H_{y}}{\partial x}-\frac{\partial H_{x}}{\partial y}-\sigma E_{z}\right), \\
\frac{\partial H_{x}}{\partial t}=\frac{1}{\mu} \cdot\left(\frac{\partial E_{y}}{\partial z}-\frac{\partial E_{z}}{\partial y}\right) \\
\frac{\partial H_{y}}{\partial t}=\frac{1}{\mu} \cdot\left(\frac{\partial E_{z}}{\partial x}-\frac{\partial E_{x}}{\partial z}\right) \\
\frac{\partial}{\mu} \cdot\left(\frac{\partial E_{x}}{\partial y}-\frac{\partial E_{y}}{\partial x}\right) .
\end{gathered}
$$

When assessing the propagation of the electromagnetic field (EM) in building materials and structures under a single storey building system, the model can be simplified to a twodimensional variant. In this case the impact of external structural elements is omitted and field distribution changes in the vertical direction are taken into account.

\section{Discretisation of the area in FDTD method}

Determination of field changes in the area of model $\Omega$ is carried out under the assumption of appropriately defined localisation of components of vectors $\boldsymbol{E}$ and $\boldsymbol{H}$. In the formulation, the difference method in the time domain takes into consideration the construction with shifted mesh for the components of the electric and magnetic field. The structure of the grid reflects the physical interpretation of electromagnetic phenomena and is compatible with the concept proposed by K. S. Yee in formulating the difference method in the time domain [6-8].

In a formulation of the FDTD method, with a three-dimensional scheme, the Yee cell is used (Fig. 1). It contains six appropriately placed component vectors of field intensity: electrical $\left\{E_{x}, E_{y}, E_{z}\right\}$ and magnetic $\left\{H_{x}, H_{y}, H_{z}\right\}$. The use of the FDTD method is based on the division of the whole analysed area into an appropriate number of cells. The FDTD schematic in the area is realised through the proper distribution of component of vectors describing intensity of the electric field $\boldsymbol{E}$ and magnetic field $\boldsymbol{H}$ within each cell (Fig. 1). Determined spatial distributions of physical quantities $\left\{E_{x}, E_{y}, E_{z}, H_{x}, H_{y}, H_{z}\right\}$ are assigned in certain points of the area $(x, y, z)$, taking into account discrete, finite size of the step of integration on the field $\left(\Delta_{x}, \Delta_{y}, \Delta_{z}\right)$. 


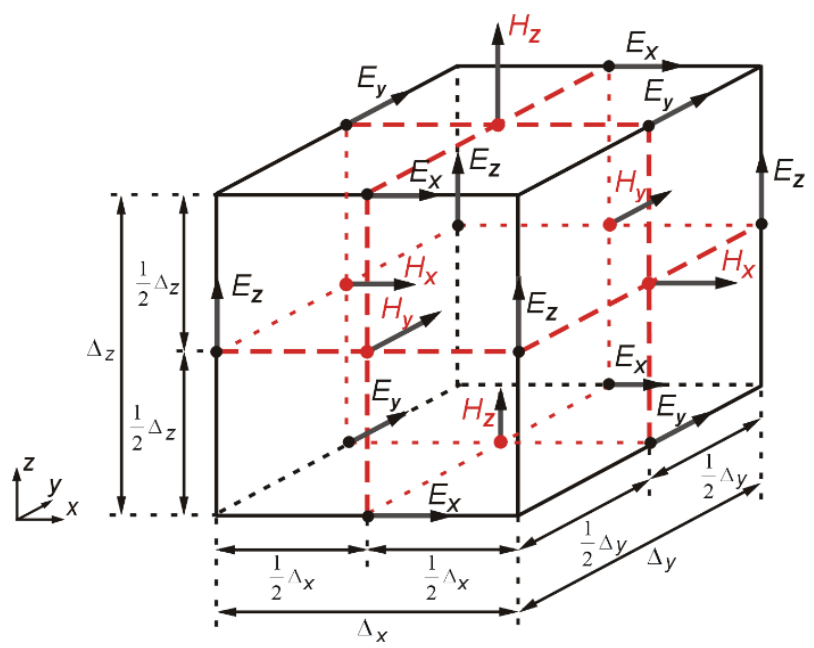

Fig. 1. The construction of the Yee cell used in the FDTD method (in 3D)

The components of the electromagnetic field are calculated in a different point in space. The $E_{x}, E_{y}, E_{z}$ components which are associated with the Yee cell are anchored in mid-points of appropriate edges, and the $H_{x}, H_{y}, H_{z}$ components - in the middle of the planes forming the sides of the cell. Appropriate component intensity vectors of the magnetic field circulating around it surround each component of the intensity vector of the electric field. The presented notation is analogical to the calculated three component intensity vectors of the magnetic field.

\section{The numerical formulation of the FDTD method}

According to the Yee scheme, iterative integration of Maxwell's equations in the time domain is based on a two-steps scheme. At selected steps $n$, time is designated to the distribution of the electric field, whereas the value of components of the vector of magnetic field intensity are calculated from the displacement at the time $\Delta_{t}$. The considered physical quantities are functions of the spatial variables $(x, y, z)$ and the time variable $(t)$.

After applying Euler's scheme of the central differences [8, 10, 12] to approximate the partial derivatives in the area and after a time, differential Eqs. (8)-(13) take the form

$$
\frac{{ }_{i, j, k}^{n+1} E_{x}-{ }_{i, j, k} E_{x}}{\Delta_{t}}=\frac{1}{\varepsilon_{i, j, k}} \cdot\left(\frac{{ }^{n+\frac{1}{2}} H_{z}-{ }_{i, j-\frac{1}{2}, k}^{n+\frac{1}{2}} H_{z}}{\Delta_{y}}-\frac{{ }_{i, j, k+\frac{1}{2}}^{n+\frac{1}{2}} H_{y}-{ }_{i, j, k-\frac{1}{2}}^{n+\frac{1}{2}} H_{y}}{\Delta_{z}}-\sigma_{i, j, k} \cdot{ }_{i, j, k}^{n+\frac{1}{2}} E_{x}\right),
$$




$$
\begin{aligned}
& \frac{{ }_{i, j, k}^{n+1} E_{y}-{ }_{i, j, k}^{n} E_{y}}{\Delta_{t}}=\frac{1}{\varepsilon_{i, j, k}} \cdot\left(\frac{{ }_{i, j, k+\frac{1}{2}}^{n+\frac{1}{2}} H_{x}-{ }_{i, j, k-\frac{1}{2}}^{n+\frac{1}{2}} H_{x}}{\Delta_{z}}-\frac{{ }_{i+\frac{1}{2}, j, k}{ }^{n+\frac{1}{2}} H_{z}-{ }_{i-\frac{1}{2}, j, k}^{n+\frac{1}{2}} H_{z}}{\Delta_{x}}-\sigma_{i, j, k} \cdot \cdot_{i, j, k} E_{y}\right), \\
& \frac{i_{i, j, k}^{n+1} E_{z}-{ }_{i, j, k}^{n} E_{z}}{\Delta_{t}}=\frac{1}{\varepsilon_{i, j, k}} \cdot\left(\frac{{ }_{i+\frac{1}{2}, j, k}{ }^{n+\frac{1}{2}} H_{y}-{ }_{i-\frac{1}{2}, j, k}^{n+\frac{1}{2}} H_{y}}{\Delta_{x}}-\frac{{ }_{i, j+\frac{1}{2}, k}^{n+\frac{1}{2}} H_{x}-{ }_{i, j-\frac{1}{2}, k}^{n+\frac{1}{2}} H_{x}}{\Delta_{y}}-\sigma_{i, j, k} \cdot{ }_{i, j, k}^{n+\frac{1}{2}} E_{z}\right), \\
& \frac{{ }_{n+\frac{1}{2}}^{n} H_{x}-{ }_{i, j, k}^{n-\frac{1}{2}} H_{x}}{\Delta_{t}}=\frac{1}{\mu_{i, j, k}} \cdot\left(\frac{{ }_{i, j, k+\frac{1}{2}}^{n} E_{y}-{ }_{i, j, k-\frac{1}{2}} E_{y}}{\Delta_{z}}-\frac{{ }_{i, j+\frac{1}{2}, k}{ }^{n} E_{z}-{ }_{i, j-\frac{1}{2}, k}^{n} E_{z}}{\Delta_{y}}\right), \\
& \frac{{ }_{i, j, k}^{n+\frac{1}{2}} H_{y}-{ }_{i, j, k}^{n-\frac{1}{2}} H_{y}}{\Delta_{t}}=\frac{1}{\mu_{i, j, k}} \cdot\left(\frac{{ }_{i+\frac{1}{2}, j, k}^{n} E_{z}-{ }_{i-\frac{1}{2}, j, k} E_{z}}{\Delta_{x}}-\frac{{ }_{i, j, k+\frac{1}{2}} E_{x}-{ }_{i, j, k-\frac{1}{2}} E_{x}}{\Delta_{z}}\right), \\
& \frac{{ }_{i, j, k}^{n+\frac{1}{2}} H_{z}-{ }_{i, j, k}^{n-\frac{1}{2}} H_{z}}{\Delta_{t}}=\frac{1}{\mu_{i, j, k}} \cdot\left(\frac{{ }_{i, j+\frac{1}{2}, k}^{n} E_{x}-{ }_{i, j-\frac{1}{2}, k}^{n} E_{x}}{\Delta_{y}}-\frac{{ }_{i+\frac{1}{2}, j, k}^{n} E_{y}-{ }_{i-\frac{1}{2}, j, k}^{n} E_{y}}{\Delta_{x}}\right) .
\end{aligned}
$$

The values ${ }_{i, j, k}^{n+\frac{1}{2}} E$ in the Eqs. (14)-(16) are approximated by the arithmetic average of solutions in following steps $n$ and $n+1$ (semi-implicit approximation) [8, 10], e.g.

$$
{ }_{i, j, k}^{n+\frac{1}{2}} E_{z}=\frac{{ }_{i, j, k}^{n+1} E_{z}+{ }_{i, j, k}^{n} E_{z}}{2} .
$$

Thus, for example, by substituting Eq. (20) into Eq. (16) we obtained the relationship given by

$$
{ }_{i, j, k}^{n+1} E_{z}=\frac{1-\frac{\sigma_{i, j, k} \cdot \Delta_{t}}{2 \varepsilon_{i, j, k}}}{1+\frac{\sigma_{i, j, k} \cdot \Delta_{t}}{2 \varepsilon_{i, j, k}}} \cdot i, j,{ }^{n} E_{z}+\frac{\frac{\Delta_{t}}{\varepsilon_{i, j, k}}}{1+\frac{\sigma_{i, j, k} \cdot \Delta_{t}}{2 \varepsilon_{i, j, k}}} \cdot\left(\frac{{ }_{i+\frac{1}{2}, j, k}^{n+\frac{1}{2}} H_{y}-{ }_{i-\frac{1}{2}, j, k}^{n+\frac{1}{2}} H_{y}}{\Delta_{x}}-\frac{{ }_{i, j+\frac{1}{2}, k}^{n+\frac{1}{2}} H_{x}-{ }_{i, j-\frac{1}{2}, k}^{n+\frac{1}{2}} H_{x}}{\Delta_{y}}\right) .
$$


With the use of molecular notation, the three-dimensional FDTD algorithm is shown in Fig. 2. To determine the value

$$
{ }_{i, j, k}^{n+1} E_{z}
$$

in the time step $n+1$ it is necessary to refer to the value of magnetic field strength calculated at the time $n+1 / 2$ and also to the value

$$
{ }_{i, j, k}^{n} E_{z} \text {. }
$$

Similarly, values for nodes for the components of the magnetic field (leapfrog) are determined $[6]$.
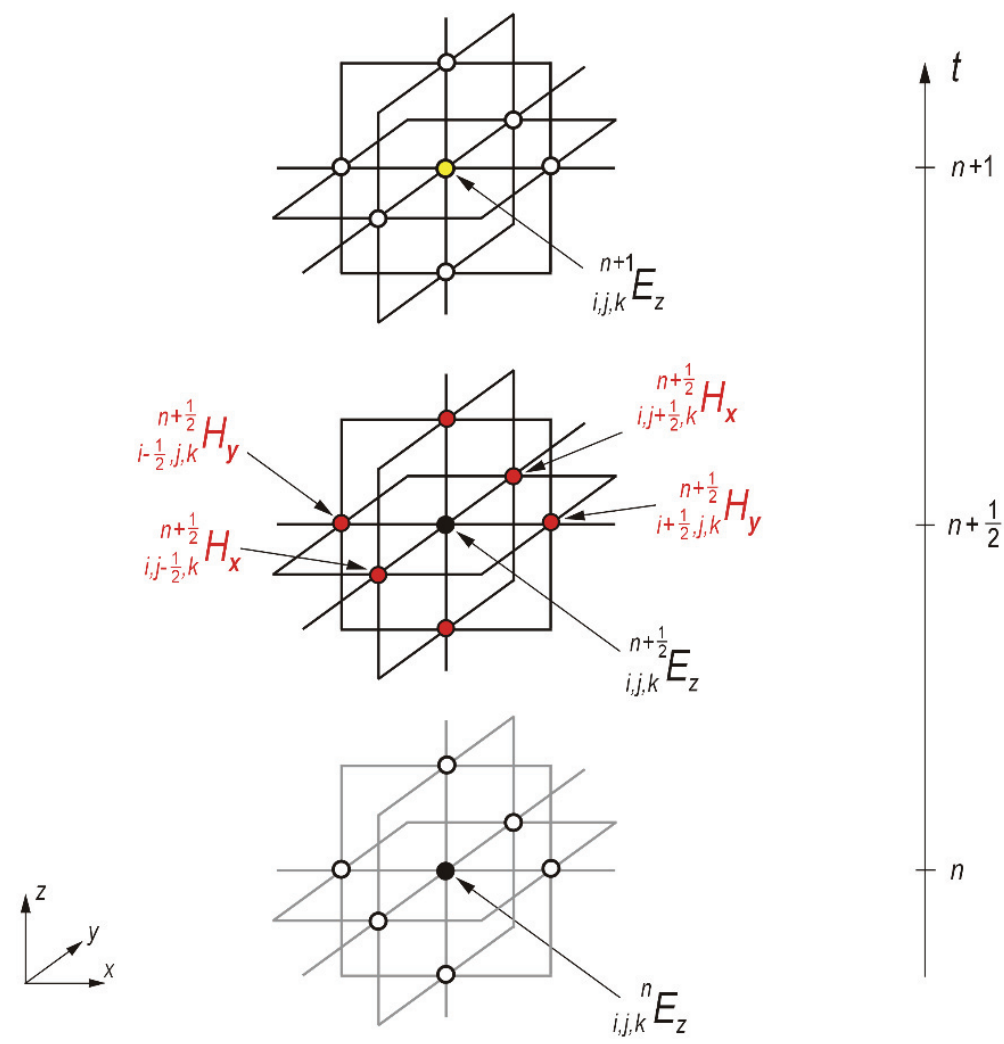

Fig. 2. Determination the values of $E_{z}$ component of the electric fields' intensity in the $n+1$ step, for Yee cell at index $i, j, k$ in the FDTD algorithm

Defining the coefficients, e.g. in Eqs. (22)-(24) describing the material properties of the Yee cells of difference mesh to write the difference equations in the FDTD algorithm in a simpler way

$$
c_{i, j, k}^{e e}=\frac{\varepsilon_{i, j, k}-\sigma_{i, j, k} \cdot \Delta_{t}}{\varepsilon_{i, j, k}+\sigma_{i, j, k} \cdot \Delta_{t}},
$$




$$
\begin{gathered}
c_{i, j, k}^{e h y}=\frac{2 \Delta_{t}}{2 \varepsilon_{i, j, k}+\sigma_{i, j, k} \cdot \Delta_{t}} \cdot \frac{1}{\Delta_{x}}, \\
c_{i, j, k}^{e h x}=\frac{2 \Delta_{t}}{2 \varepsilon_{i, j, k}+\sigma_{i, j, k} \cdot \Delta_{t}} \cdot \frac{1}{\Delta_{y}} .
\end{gathered}
$$

Substitute coefficients are introduced to describe the equations for the components of the magnetic field. For example, the component

$$
{ }_{i, j, k}^{n+\frac{1}{2}} H_{z}
$$

that was defined by Eq. (19) used the coefficients given by

$$
\begin{gathered}
c_{i, j, k}^{h h}=\frac{2 \mu_{i, j, k}-\Delta_{t}}{2 \mu_{i, j, k}+\Delta_{t}}, \\
c_{i, j, k}^{h e y}=\frac{\Delta_{t}}{\mu_{i, j, k}} \cdot \frac{1}{\Delta_{x}}, \\
c_{i, j, k}^{h e x}=\frac{\Delta_{t}}{\mu_{i, j, k}} \cdot \frac{1}{\Delta_{y}} .
\end{gathered}
$$

Using similar conversions of Eqs. (14)-(19) the remaining coefficients:

$$
c_{i, j, k}^{e h z}, c_{i, j, k}^{h e z}
$$

are obtained. When using a cubic mesh $\Delta=\Delta_{x}=\Delta_{y}=\Delta_{z}$, a description of each cells of the mesh is reduced to two coefficients determined for the following coefficients appearing in the model material

$$
\begin{aligned}
& c_{i, j, k}^{e h}=c_{i, j, k}^{e h x}=c_{i, j, k}^{e h y}=c_{i, j, k}^{e h z}, \\
& c_{i, j, k}^{h e}=c_{i, j, k}^{h e x}=c_{i, j, k}^{h e y}=c_{i, j, k}^{h e z} .
\end{aligned}
$$

The included assumptions and applying coefficients allow to simplify the notation of difference equations in the FDTD algorithm

$$
\begin{aligned}
{ }_{i, j, k}^{n+1} E_{x} & =c_{i, j, k}^{e e}{ }^{\cdot i, j, k}{ }^{n} E_{x}+ \\
& +c_{i, j, k}^{e h} \cdot\left(\begin{array}{c}
n+\frac{1}{2} \\
i, j+\frac{1}{2}, k
\end{array} H_{z}-{ }_{i, j-\frac{1}{2}, k}^{n+\frac{1}{2}} H_{z}-{ }_{i, j, k+\frac{1}{2}}^{n+\frac{1}{2}} H_{y}+{ }_{i, j, k-\frac{1}{2}}^{n+\frac{1}{2}} H_{y}\right),
\end{aligned}
$$




$$
\begin{aligned}
& { }_{i, j, k}^{n+1} E_{y}=c_{i, j, k}^{e e} \cdot{ }_{i, j, k}^{n} E_{y}+
\end{aligned}
$$

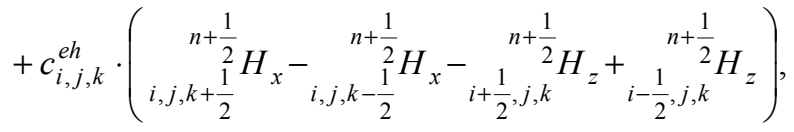

$$
\begin{aligned}
& { }_{i, j, k}^{n+1} E_{z}=c_{i, j, k}^{e e} \cdot{ }_{i, j, k}^{n} E_{z}+ \\
& +c_{i, j, k}^{e h} \cdot\left(\begin{array}{c}
{ }^{n+\frac{1}{2}} H_{y}-{ }_{i-\frac{1}{2}, j, k}^{n+\frac{1}{2}} H_{y}-{ }_{i, j+\frac{1}{2}, k}^{n+\frac{1}{2}} H_{x}+{ }_{i, j-\frac{1}{2}, k}^{n+\frac{1}{2}} H_{x} \\
i+\frac{1}{2}
\end{array}\right), \\
& { }_{i, j, k}^{n+\frac{1}{2}} H_{x}=c_{i, j, k}^{h h} \cdot{ }_{i, j, k}^{n-\frac{1}{2}} H_{x}+ \\
& +c_{i, j, k}^{h e} \cdot\left({ }_{i, j, k+\frac{1}{2}}^{n} E_{y}-{ }_{i, j, k-\frac{1}{2}}^{n} E_{y}-{ }_{i, j+\frac{1}{2}, k}^{n} E_{z}+{ }_{i, j-\frac{1}{2}, k}^{n} E_{z}\right) \text {, } \\
& { }_{i, j, k}^{n+\frac{1}{2}} H_{y}=c_{i, j, k}^{h h} \cdot{ }_{i, j, k}^{n-\frac{1}{2}} H_{y}+ \\
& +c_{i, j, k}^{h e} \cdot\left({ }_{i+\frac{1}{2}, j, k}^{n} E_{z}-{ }_{i-\frac{1}{2}, j, k}^{n} E_{z}-{ }_{i, j, k+\frac{1}{2}}^{n} E_{x}+{ }_{i, j, k-\frac{1}{2}}^{n} E_{x}\right), \\
& { }_{i, j, k}^{n+\frac{1}{2}} H_{z}=c_{i, j, k}^{h h} \cdot{ }_{i, j, k}^{n-\frac{1}{2}} H_{z}+ \\
& +c_{i, j, k}^{h e} \cdot\left({ }_{i, j+\frac{1}{2}, k}^{n} E_{x}-{ }_{i, j-\frac{1}{2}, k}^{n} E_{x}-{ }_{i+\frac{1}{2}, j, k}^{n} E_{y}+{ }_{i-\frac{1}{2}, j, k}^{n} E_{y}\right) .
\end{aligned}
$$

Final Eqs. (30)-(35) indicate that the formulation of the FDTD algorithm leads to receiving the explicit scheme in which the matrix $\boldsymbol{A}$ describing the analysed problem is not formed. The construction of the numerical model requires only writing the table of coefficients $\left\{c_{i, j, k}^{e e}\right.$, $\left.c_{i, j, k}^{e h x}, c_{i, j, k}^{e h y}, c_{i, j, k}^{\text {ehz }}, c_{i, j, k}^{h h}, c_{i, j, k}^{h e x}, c_{i, j, k}^{h e y}, c_{i, j, k}^{h e z}\right\}$ describing individual Yee cells.

\section{Analysed model}

In the macroscopic approach, clay hollow bricks are classified as complex building structures. In such circumstances the assessment of the EM field distribution may only be performed by usage of numerical analysis. The object of analysis was area with a single $(0.12 \mathrm{~m})$ and double-layered wall $(0.24 \mathrm{~m})$ made of clay brick with 18 vertical holes (Fig. 3a). The dimensions of one brick were: $0.065 \mathrm{~m}$ of height $(h), 0.12 \mathrm{~m}$ - width $(w), 0.25 \mathrm{~m}$ - length $(l)$ (Fig. 3b) [12].

It should be noted, that in an electrical sense, the analysed problem is limited to the analysis of the porous elements because: 
1) there is a periodic arrangement of materials,

2) the electromagnetic wave propagates through a multi-layered structure contained air and a non-ideal dielectric (the clay mass),

3 ) the dimensions of the brick and holes (with the one of the analysed frequency of $f=2.4 \mathrm{GHz}$ ) are comparable to the length of the EM wave propagating in air $\lambda_{a}=0.125 \mathrm{~m}$ (Fig. 3b).

For all analysed cases the constant value of the relatively electric permittivity was assumed $\varepsilon_{r}=4.44[1,4,5]$. The conductivity was changed in the range $\sigma \in\langle 0.01,0.2\rangle \mathrm{S} / \mathrm{m}[1,4,5,12]$. The Fig. $3 \mathrm{~b}$ shows also the electrical dimensions of clay hollow brick at $f=2.4 \mathrm{GHz}$, where $\lambda_{b}=0.0593 \mathrm{~m}$ is the length of the wave in a clay material.

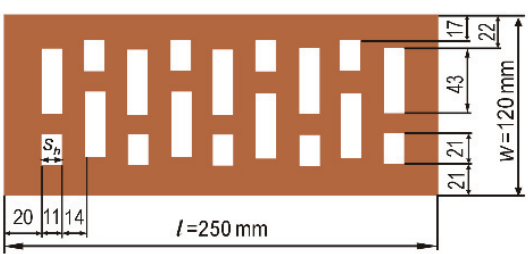

(a)

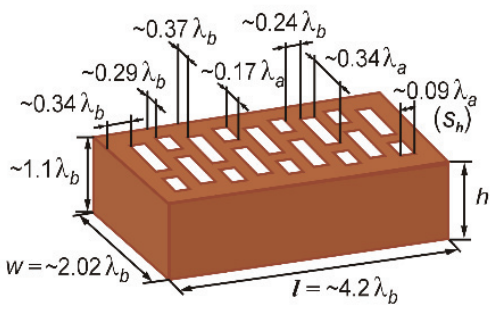

(b)

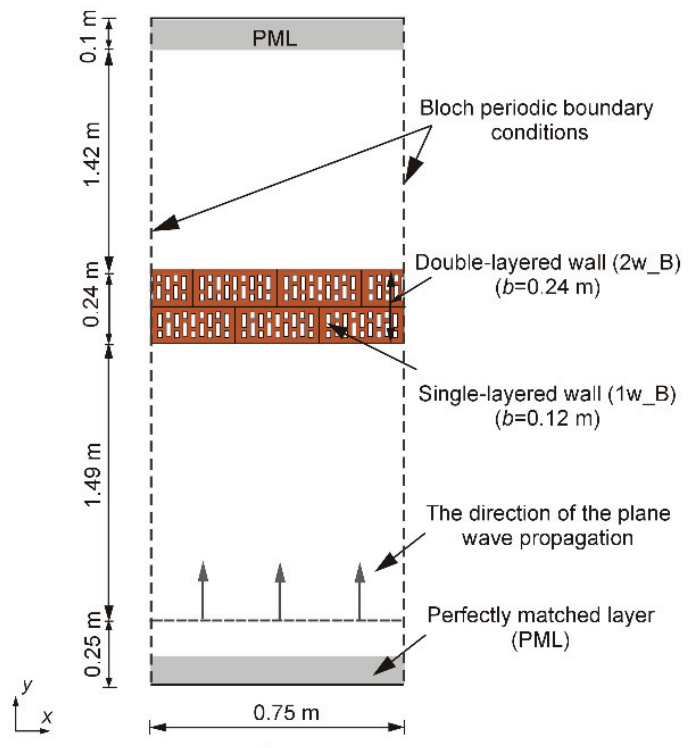

(c)

Fig. 3. Analysed problem: (a) the geometry of brick; (b) electrical sizes of the brick; (c) the geometry and numerical conditions of the examined area with the wall [12]

It should be pointed out, that after the drying and hardening processes some parts of the brick might have an untypical size. Additionally, for this reason the influence of a different relative volume of the clay mass on the values of electric field was analysed. This factor, by changing the size of air holes $\left(s_{h}\right)$ was modified (Table 1$)$.

Table 1. Percentage of the ceramic volume inside the brick with different size of the holes

\begin{tabular}{c|c|c}
\hline $\begin{array}{c}\text { Geometric size } \\
\left(\boldsymbol{s}_{\boldsymbol{h}}\right)[\mathbf{m}]\end{array}$ & $\begin{array}{c}\text { Electric size } \\
\left(\boldsymbol{s}_{\boldsymbol{h}}\right)[\mathbf{m}]\end{array}$ & $\begin{array}{c}\text { Relative volume of the clay mass } \\
\text { in the brick }\left(\boldsymbol{V}_{\% \boldsymbol{m}}\right)\end{array}$ \\
\hline 0.005 & $0.040 \lambda_{a}$ & $90.4 \%$ \\
\hline 0.007 & $0.056 \lambda_{a}$ & $86.5 \%$ \\
\hline
\end{tabular}




\begin{tabular}{l|l|l}
\hline 0.009 & $0.072 \lambda_{a}$ & $82.7 \%$ \\
\hline 0.011 & $0.088 \lambda_{a}$ & $78.8 \%$ \\
\hline 0.013 & $0.104 \lambda_{a}$ & $75.0 \%$ \\
\hline 0.015 & $0.120 \lambda_{a}$ & $71.2 \%$ \\
\hline 0.017 & $0.136 \lambda_{a}$ & $67.3 \%$ \\
\hline 0.019 & $0.152 \lambda_{a}$ & $63.5 \%$ \\
\hline
\end{tabular}

The typical average size of air holes in this kind of brick amounts to $0.011 \mathrm{~m}$. Percentage of the total brick volume inside the brick with different size of the holes was calculated by using the ratio

$$
V_{\% m c}=\frac{V_{c}-V_{d}}{V_{c}} \cdot 100 \%,
$$

where $V_{d}$ is the volume of all holes inside the brick, $V_{c}=h \cdot w \cdot l$.

\section{Construction of numerical model}

To determine the distribution of the electromagnetic field the previously described FDTD method was used. The stability of the time marching explicit scheme requires satisfying the Courant-Friedrichs-Lewy (CFL) condition [6]. It defines the dependence between the minimum value of the integration step in the time domain $\Delta_{t}$ and the maximum size of the Yee cell. In analysed models $\Delta_{x}=\Delta_{y}=0.0016667 \mathrm{~m}$ and guaranteed the fulfilment of this condition. Due to this, the length of the wave in the air $\left(\lambda_{a}\right)$ corresponded to 75 Yee cells. The EM field is excited in a region far away of the wall. The source of the field was a sinusoidal oscillating plane wave with also $f=2.4 \mathrm{GHz}$ and $f=5 \mathrm{GHz}$ (typical values using by Wi-Fi). The absorption of the incident and reflected waves are obtained using PML boundary conditions (perfectly matched layer) $[6,7]$. These were entered in the outside areas, perpendicular to the equiphase surface (Fig. 3c).

The main aim of the analysis was to determine the value of the electric field in the area behind the wall. It was assumed that the crosswise dimensions of the wall are significantly larger than: the dimensions of the brick and the length of the wave. Incidents at the edges of the wall were not taken into account and then Bloch's periodic boundary conditions were assumed (in the edges perpendicular to the $O x$ axis) [7]. For this reason, the length of the wall could be reduced to three bricks (Fig. 3c).

\section{Results of analysis}

The FDTD method allows to obtain instantaneous field images. For this reason, it was necessary to develop an additional algorithm. The aim of the mentioned algorithm was to prepare the map describing the maximum values of the field in the processing sequence of 
instantaneous field distribution, calculated at regular intervals. The formulated algorithm and developed software tool to determine the maximum values of the presented component field enabled to perform the analysis of the discussed issue and also prepared below Figs. 4-7.

The received results of calculation are shown as the steady state of the electromagnetic field distribution that has been achieved. Fig. 4 presents, in the $X Y$ plane, distributions of relative values of maximum $E_{z}$ component in the area behind the wall, which were obtained for the most commonly using value of the conductivity of brick $(0.02 \mathrm{~S} / \mathrm{m})$. During the passing over the different areas of bricks, the local changes of the wave velocity cause the appearance of the instantaneous images of the field. The presented effect is particularly visible during the evaluation of phenomenon which occurred at $f=5 \mathrm{GHz}$.

Reducing the length of electromagnetic wave causes the appearance of a greater number of maxima and minima. They are local and sometimes their values are higher by approximately $20 \%$ than the value obtained at a frequency $2.4 \mathrm{GHz}$. The same effect is in the case of the minima, which, however, have a lower value (approximately $20 \%$ ).

The indicated phenomenon is connected with the interaction of an electromagnetic wave on the boundary of medium, inside the clay hollow bricks, which multiples reflections from edges of the holes. This fact causes local increase and decrease of the values of the electric field behind the analysed wall.

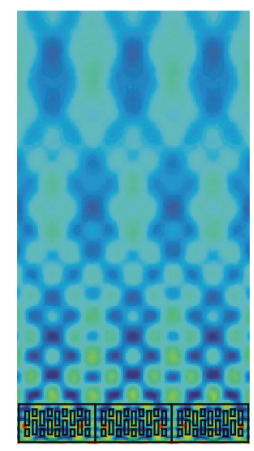

(a)

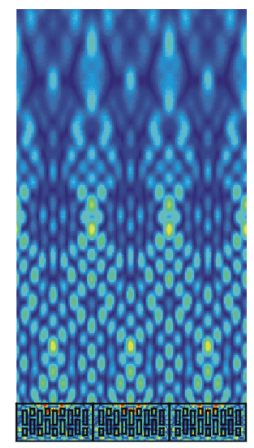

(b)

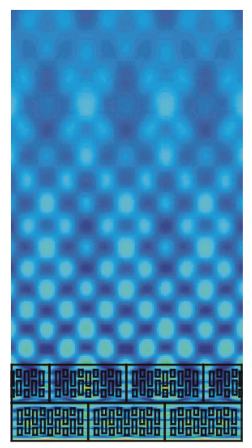

(c)

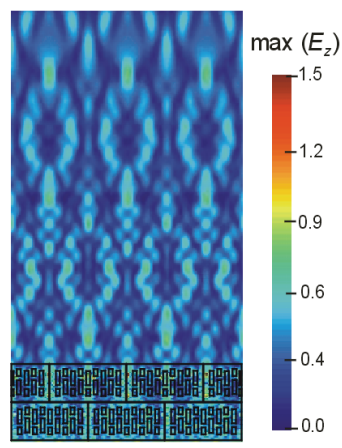

(d)

Fig. 4. The distribution of the relative maximum values of $E_{z}$ component: (a) single-layered wall, $f=2.4 \mathrm{GHz}$, (model: $1 \mathrm{w} \_2.4$ ); (b) single-layered wall, $f=5 \mathrm{GHz},(1 \mathrm{w}$ 5); (c) double-layered wall, $f=2 . \overline{4} \mathrm{GHz},\left(2 \mathrm{w} \_2.4\right)$; (b) double-layered wall, $f=5 \mathrm{GHz},\left(2 \mathrm{w} \_5\right)$

The changes of the relative value of the maximum $E_{z}$ component depending on the value of conductivity of the material and the size of the holes inside the brick $\left(s_{h}\right)$ for a single and double-layered wall were shown in Fig. $5(f=2.4 \mathrm{GHz})$ and Fig. $6(f=5 \mathrm{GHz})$.

In the analysis of variants of the material with relatively low losses $\sigma \in\langle 0,0.05\rangle \mathrm{S} / \mathrm{m}$ charts present complex waveforms with a visible minimum at $s_{h}=0.017 \mathrm{~m}\left(1 \mathrm{w} \_2.4\right)$, $s_{h}=0.009 \mathrm{~m}\left(2 \mathrm{w}_{2} 2.4\right)$. This indicates that the lack of monotonicity is much more visible in models at higher frequency $(f=5 \mathrm{GHz})$. For example in the model with a double-layered wall (2w_5) two minima: $s_{h}=0.013 \mathrm{~m}, s_{h}=0.019 \mathrm{~m}$ were observed. The observed effects should be attributed to the complexity of the phenomena field using wave propagation in porous material. 


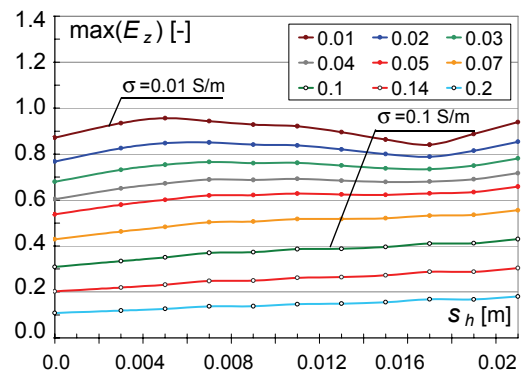

(a)

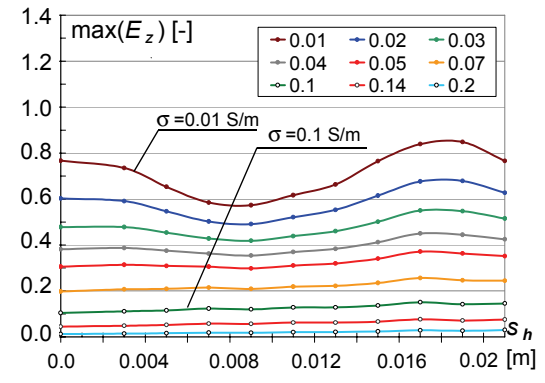

(b)

Fig. 5. The relative maximum values of $E_{z}$ component behind: (a) single-layered wall, $f=2.4 \mathrm{GHz}$ (1w_2.4); (b) double-layered wall, $f=2.4 \mathrm{GHz}(2 \mathrm{w} 2.4)$

A significant lossiness of the material $(\sigma \in\langle 0.1,0.02\rangle \mathrm{S} / \mathrm{m})$ causes that the waveform characteristics are similar to the variations of the homogeneous losses material. This effect is especially visible at $f=2.4 \mathrm{GHz}$, at a range of $\sigma \in\langle 0.1,0.02\rangle \mathrm{S} / \mathrm{m}$ ) for the both single-layered wall and double-layered wall, as shown in Fig. 5. Increasing the size of holes (air) by decreasing non-ideal dielectric reduces attenuation.

The porous structure of the analysed bricks in an electromagnetic sense influences the occurrence of multiple reflections at the air-brick border. The number and size of the holes inside the clay hollow brick results in temporary changes of field image in the area close behind the wall. A local change of the speed of the wave while passing through various parts of the brick is depicted in the distribution of the field and occurrence of interference. A higher percentage of clay material in the wall causes smaller distortion of the wave front in the area behind the wall.

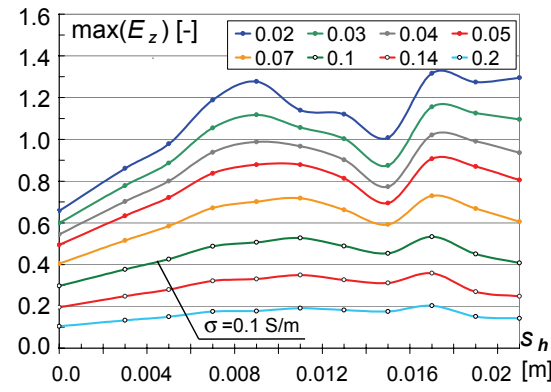

(a)

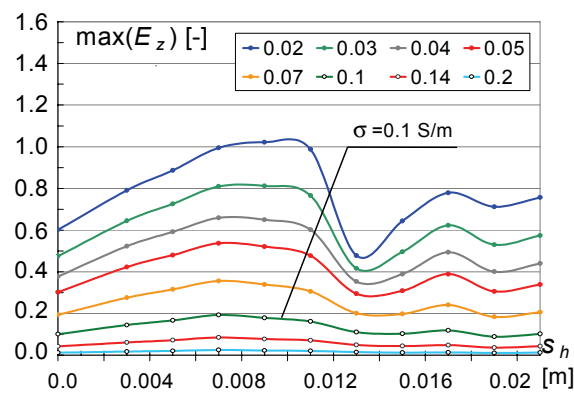

(b)

Fig. 6. The relative values of $\max \left(E_{z}\right), f=5 \mathrm{GHz}$ for model: (a) $1 \mathrm{w} \_5$; (b) $2 \mathrm{w} \_5$

In Figs. 7-8 have been presented comparing the growth of the maximum of the relative values of $E_{z}$ component depending on the percentage of the ceramic mass analysed models in three sub-ranges of conductivity. These include the range of typical changes in this $\sigma \in\langle 0.02,0.09\rangle \mathrm{S} / \mathrm{m}$ for frequencies used in Wi-Fi.

Regardless of the analysed model with the wall made of bricks, the largest difference in the values of the presented components of the electric field were observed in the range of 
$\sigma \in\langle 0.04,0.09\rangle \mathrm{S} / \mathrm{m}$. Whereas, the smallest span values of $\max \left(E_{z}\right)$ are visible for $\sigma \in\langle 0.02,0.03\rangle \mathrm{S} / \mathrm{m}$, similarly as for $\sigma \in\langle 0.03,0.04\rangle \mathrm{S} / \mathrm{m}$ but in this range of conductivity the values of the electric field are lower because of growth of losses in the material.

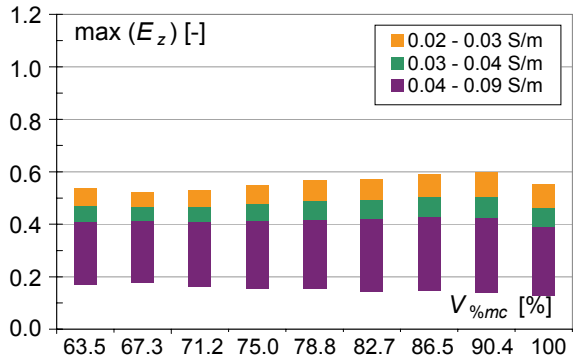

(a)

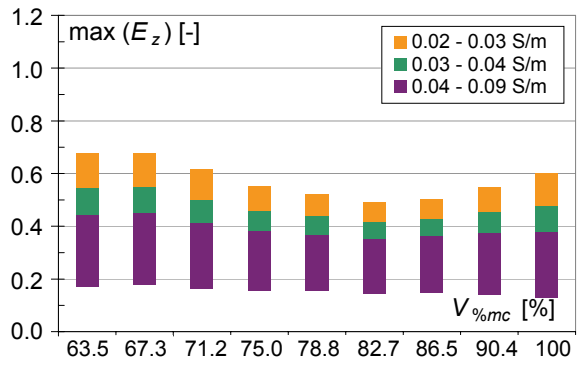

(b)

Fig. 7. Comparing the changes of relative maximum values of $E_{z}$ component dependent on the relative volume of the clay in the brick $V_{\% \mathrm{mc}}$ at $f=2.4 \mathrm{GHz}$

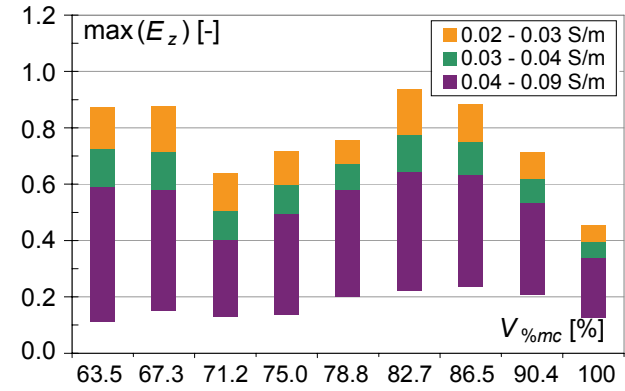

(a)

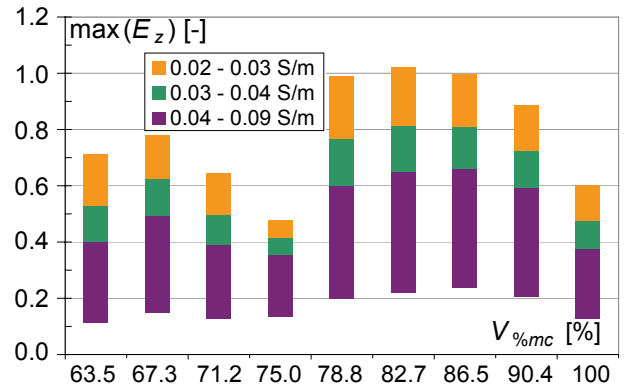

(b)

Fig. 8. Comparing the changes of $\max \left(E_{z}\right)$ dependent on $V_{\% \mathrm{mc}}$ at $f=5 \mathrm{GHz}$

It was also observed that at the higher frequencies $(f=5 \mathrm{GHz})$ the range of changes of the values $E_{z}$ component in analysed sub-ranges is almost double higher than in model at $f=2.4 \mathrm{GHz}$.

In order to verify the results obtained using the method described, the FDTD analysis was also made using the method of FEM (Finite Element Method) [11]. It was used to model a single-layer wall, chosen for three values of the size of the drill. In Fig. 9, the results show that the values of the intensity field decreases with increasing conductivity. The greatest differences are in the values of the order of 5\%. This is an acceptable mistake and the resulting differences of the both used methods. Boundary conditions (periodicity and PML) were unchanged. The differences are due to the mesh differential applied. In the FEM method it was possible to use mesh differential composed of triangular elements and its local compression. 


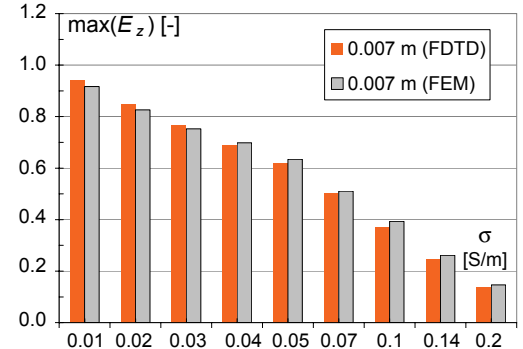

(a)

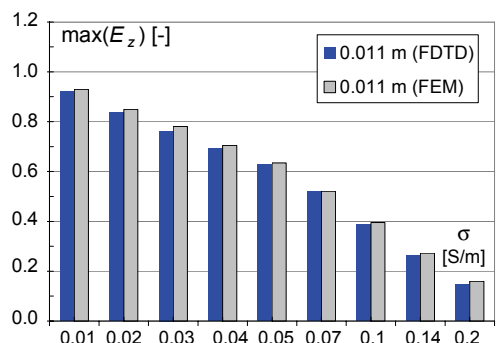

(b)

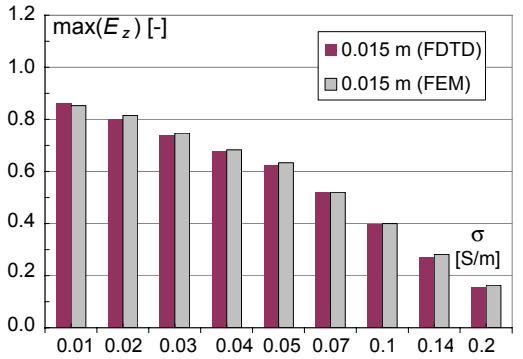

c)

Fig. 9. Comparing the changes of maximum values of $E_{z}$ component behind single-layered wall, $f=2.4 \mathrm{GHz}$ for FDTD and FEM method: (a) $s_{h}=0.007 \mathrm{~m}$; (b) $s_{h}=0.011 \mathrm{~m}$; (c) $s_{h}=0.015 \mathrm{~m}$

\section{Conclusions}

The contemporary development of wireless communication technology demands the detailed analysis of the phenomena connected with the propagation of electromagnetic waves inside the buildings. The described charts and their graphical presentation indicate complex wave phenomena that occur during wave propagation through the wall made of clay hollow brick, which are treated as electrically porous material. Various constructions of walls, and variability of material parameters have to be individually examined because e.g. the increase in the loss of brick, the main role in the phenomena process has the attenuation of the wave at crossing over this kind of complex dielectric. This fact causes that it is impossible find the dependence, which will help in the evaluation of other variants of the hollow brick (without prior numerical analysis).

In the case of a macroscopic analysis of an electromagnetic field inside buildings it is necessary to homogenise and to simplify the construction and to homogenise the material properties. This approach is imposed by the limitation of computer's calculation possibilities, taking into account the numerical modelling of a complex structure. The obtained values of electric field intensity allow to determining the attenuation coefficient for different variants of the walls.

Additionally it was proven that the large range of changeability of the value of the conductivity used to numerical analysis of these kinds of building structures might have the influence 
on authenticity of the results. The analysis of the obtained results have indicated that each wall on the way of the EM wave, especially the one made of hollow clay bricks cause the decreased intensity of EM and its irregularity.

The paper describes the use of a FDTD method of calculation of EM field distribution. The formulas presented in this paper require only a record of an array of coefficients that characterise individual Yee cells. It should be noted, that in order to calculate more complex structures the presented method could be extended by the possibility of creating more dense mesh, by using the mesh locally thickened. Also, it is possible to develop the described method of additional recording the maximum values of field components obtained as a result of processsing of many instantaneous distributions. The indicated solution avoids the use of an additionnally developed algorithm.

It should be pointed out, that one of the advantages of the described FDTD method leads to an explicit scheme in which a matrix $\boldsymbol{A}$, describing the problem, is not formed. The indicated approach is used in the FDFD method and due to the large data set requires an additional method connected with the packing matrix.

\section{Acknowledgements}

This work was prepared under scientific work S/WE/1/13 and supported by the Polish Ministry of Science and Higher Education.

\section{References}

[1] Stavrou S., Saunders S.R., Review of constitutive parameters of building material, IEEE Transactions Antennas and Propagation, Twelfth International Conference on (Conf. Publ. no. 491), (ICAP 2003), pp. 211-215 (2003).

[2] Tan S.Y., Tan Y., Tan H.S., Multipath delay measurements and modeling for interfloor wireless communications, IEEE Transactions on Vehicular Technology, vol. 49, no. 4, pp. 1334-13341 (2000).

[3] Choroszucho A., The analysis of the diameter of reinforcement, spacing between bars and the electrical parameters of the concrete on the values of the electric field intensity, Przegląd Elektrotechniczny, vol. 90, no. 2, pp. 156-160 (2014).

[4] Choroszucho A., Butryło B., The numerical analysis of the influence the incidence angle of the plane wave on the values of the electric field intensity inside the models with the complex construction of a wall, Przegląd Elektrotechniczny, vol. 90, no. 12, pp. 21-24 (2014).

[5] Pinhasi Y., Yahalom A., Petnev S., Propagation of ultra wide-band signals in lossy dispersive media, IEEE International Conference on Microwaves, Communications, Antennas and Electronic Systems, COMCAS, pp. 1-10 (2008).

[6] Taflove A., Hagness S.C., Computational electrodynamics, the finite-difference time-domain method, Boston, Artech House (2005).

[7] Oskooi A.F., Roundyb D., Ibanescua M. et al., MEEP: A flexible free-software package for electromagnetic simulations by the FDTD method, Computer Physics Communications, vol. 181, no. 3, pp. 687-702 (2010).

[8] Elsherbeni A. Z., Demir V., The finite-difference time-domain method for electromagnetics with MATLAB Simulations, SciTech Publishing (2009).

[9] Butryło B., Parallel computations of electromagnetic fields in models with dispersive materials (in Polish), Oficyna Wydawnicza Politechniki Białostockiej (2012).

[10] Bronsztejn I.N., Siemiendiajew K.A., Mathematics. An encyclopedic guide (in Polish), PWN (2004).

[11] Comsol Multiphysics user's guide, Comsol AB (2009).

[12] Choroszucho A., An analysis of the electromagnetic waves propagation in construction elements with a complex structure in the range of wireless communication (in Polish), Ph.D dissertation, Białystok (2014). 